\title{
Cardiac Computed Tomography for the Diagnosis of Coronary Artery Atherosclerosis
}

\author{
Ryotaro Wake ${ }^{1,2,3^{*}}$, Hidetaka Iida ${ }^{1,2}$, Hirohito Ogata ${ }^{2}$, Hiroaki Takeshita ${ }^{2,3}$, Takanori Kusuyama ${ }^{2}$, \\ Hiroaki Kohno $^{2}$, Shinichi Shimodozono ${ }^{1,2}$, Kenei Shimada ${ }^{3}$, Minoru Yoshiyama ${ }^{3}$ \\ ${ }^{1}$ Department of Cardiology, Tsukazaki Memorial Hospital, Himeji, Japan; ${ }^{2}$ Department of Cardiology, Tsukazaki Hospital, Himeji, \\ Japan; ${ }^{3}$ Department of Internal Medicine and Cardiology, Osaka City University Graduate School of Medicine, Osaka, Japan. \\ Email: *ryotaro_wake@yahoo.co.jp
}

Received December $18^{\text {th }}, 2012$; revised February $12^{\text {th }}, 2013$; accepted March $13^{\text {th }}, 2013$

\begin{abstract}
Coronary artery disease (CAD) is a leading cause of mortality and morbidity in developed countries, although percutaneous coronary intervention and coronary artery bypass grafting have developed recently. Appropriate diagnosis will improve the prevention, treatment, and care of all patients. We could diagnose only calcification in the coronary arteries with the past computed tomography. Recently, multislice computed tomography has been already accepted as an efficient non-invasive tool for the detection of coronary artery stenosis. We get to estimate the coronary artery stenosis with cardiac computed tomography. We discuss the usefulness of cardiac computed tomography for the risk stratification of coronary artery atherosclerosis.
\end{abstract}

Keywords: Cardiac CT; Coronary Artery Atherosclerosis

\section{Introduction}

Coronary artery disease (CAD) is a leading cause of mortality and morbidity in developed countries [1]. CAD is a common and sometimes disabling disorder, although percutaneous coronary intervention and coronary artery bypass grafting have developed. Medical doctors need to prevent acute coronary syndrome in the stage of no significant stenotic coronary artery atherosclerosis. The development of non-invasive cardiac imaging tools (particularly, cardiac computed tomography, echocardiography and so on) for the diagnostic and prognostic assessments of patients is evolving evidence base for various treatment strategies. Cardiac catheterization is golden standard for the diagnosis of CAD. Although the risk of adverse events for invasive coronary angiography is generally considered to be low, potential life-threatening complications can arise, including not only coronary artery dissection, but also arrhythmia, stroke, hemorrhage, myocardial infarction (MI), and death [2]. Computed tomography (CT) imaging was introduced in 1972 [3]. The ability to obtain cross-sectional images of the computer-assisted tomography, Sir Geoffrey N. Hounsfield and Allan M. Cormack were awarded the Nobel prize in Medicine in 1979. Moreover, cardiac CT has been developing.

\footnotetext{
*Corresponding author.
}

Although coronary $\mathrm{CT}$ angiography presently cannot be considered a routine replacement for invasive coronary angiography, because the diagnostic accuracy of coronary CT angiography is not greater than that of invasive coronary angiography, cardiac CT is useful for diagnosis of coronary artery disease non-invasively.

We discuss the usefulness of cardiac computed tomography for the risk stratification of coronary artery atherosclerosis.

\section{The Diagnosis of Coronary Artery Disease with Cardiac CT}

Table 1 shows that the likelihood of ischemia due to CAD. We estimate the likelihood of ischemia in the medical history, physical examination, and cardiac biomarker measurements in patients with symptoms suggestive of acute coronary syndrome. Invasive coronary angiography is more appropriate than $\mathrm{CT}$ angiography for patients with a high pretest likelihood of CAD. CT angiography is appropriate for stable patients with acute chest pain [4]. A recent guideline reports several appropriate indications for coronary CT angiography. Firstly, they are symptomatic patients with intermediate likelihood of CAD but in whom stress testing, including in electrocardiogram, echocardiogram, and scintigram is not possible or the result of stress testing is equivocal or uninterpretable with acute chest pain. Secondly, they are 
Table 1. We show the likelihood that signs and symptoms represent acute coronary syndrome secondary to CAD. CAD indicates coronary artery disease; CK-MB: MB fraction of creatine kinase; ECG: electrocardiogram, MI: myocardial infarction, MR: mitral regurgitation. TnI: troponin I, TnT: troponin T (6).

\begin{tabular}{|c|c|c|c|}
\hline & High likelihood & Intermediate likelihood & Low likelihood \\
\hline Feature & Any of the following: & $\begin{array}{l}\text { Absence of high-likelihood features } \\
\text { and presence of any of the } \\
\text { following: }\end{array}$ & $\begin{array}{l}\text { Absence of high- or } \\
\text { intermediate-likelihood features } \\
\text { but may have the following: }\end{array}$ \\
\hline \multirow{2}{*}{ History } & $\begin{array}{l}\text { Chest or left arm pain or discomfort } \\
\text { as chief symptom reproducing prior } \\
\text { documented angina }\end{array}$ & $\begin{array}{l}\text { Chest or left arm pain or discomfort } \\
\text { as chief symptom } \\
\text { Age greater than } 70 \text { years }\end{array}$ & $\begin{array}{c}\text { Probable ischemic symptoms in } \\
\text { absence of any of the intermediate } \\
\text { likelihood characteristics }\end{array}$ \\
\hline & $\begin{array}{l}\text { Known history of CAD, } \\
\text { including MI }\end{array}$ & $\begin{array}{c}\text { Male sex } \\
\text { Diabetes mellitus }\end{array}$ & Recent cocaine use \\
\hline Physical examination & $\begin{array}{c}\text { Transient MR murmur, hypotension, } \\
\text { diaphoresis, pulmonary edema, } \\
\text { or rales }\end{array}$ & Extracardiac vascular disease & $\begin{array}{l}\text { Chest discomfort reproduced by } \\
\text { palpitation }\end{array}$ \\
\hline \multirow[t]{2}{*}{ ECG } & \multirow{2}{*}{$\begin{array}{l}\text { New, or presumably new, Transient } \\
\text { ST-segment deviation ( } 1 \mathrm{~mm} \text { or } \\
\text { greater) or T-wave inversion in } \\
\text { multiple precordial leads }\end{array}$} & Fixed Q waves & $\begin{array}{c}\text { T-wave flattering or inversion less } \\
\text { than } 1 \mathrm{~mm} \text { in leads with dominant } \\
\mathrm{R} \text { waves }\end{array}$ \\
\hline & & $\begin{array}{c}\text { ST depression } 0.5 \text { to } 1 \mathrm{~mm} \text { or } \\
\text { T-wave inversion greater than } 1 \mathrm{~mm}\end{array}$ & Normal ECG \\
\hline Cardiac markers & $\begin{array}{c}\text { Elevated cardiac TnI, TnT, or } \\
\text { CK-MB }\end{array}$ & Normal & Normal \\
\hline
\end{tabular}

patients with acute chest pain and intermediate likelihood of CAD but absence of ECG changes and normal myocardial enzyme levels (Figures 1 and 2) [5,6].

We often careful for some disadvantages of cardiac CT. Firstly, we should be careful for the renal function, because contrast nephropathy sometimes develops. And, we should be careful not to use cardiac CT for less than 50 years old women, because of radiation exposure. Next, we should confirm the patient's contrast allergy. Then, we should be careful for the calcified coronary arteries in hemodialysis and elderly patients with plain $\mathrm{CT}$, because it is difficult to diagnose CAD in the patients with many calcified coronary arteries. Lastly, we control the patient's heart rate in less than 60 beats per minute with beta blocker, especially for arrhythmia patients.

The best approaches to the care of CAD, improving not only the efficacy and safety of treatments, but also the cost.

The stratification of pretest probability of CAD are shown in Table 1.

The age, sex, and symptom groups were then used to classify each patient before diagnostic testing into 1 of 32 subsets associated with a specific likelihood for significant angiographic coronary artery disease. These preset likelihoods which are based upon a previously published review of 28948 patients in the medical literature $[7,8]$ are listed in Table 2.

Very low pretest probability is less than $5 \%$ pretest probability of CAD.
Low pretest probability is between $5 \%$ and $10 \%$ pretest probability of CAD.

Intermediate pretest probability is between $10 \%$ and $90 \%$ pretest probability of CAD. High pretest probability is more than $90 \%$ preset probability of CAD.

The Duke's treadmill score (Bruce protocol) is calculated as follows: duration of exercise in minutes $(5 \times$ the maximal ST segment deviation during or after exercise, in millimeters $)-(4 \times$ the treadmill angina index $)$.

The angina index has a value of 0 : if the patient had nonlimiting angina, 1 : if exercise angina occurred, and 2: if angina was the reason the patient stopped exercising. The score had a range from -25 (highest risk) to +15 (lowest risk).

The outpatients had treadmill scores indicating low risk $(\geq+5)$ and their four-year survival rate was $99 \%$ (average annual mortality rate, $0.25 \%$ ). The outpatients had scores indicating high risk $(<-10)$ and their four-year survival rate was $79 \%$ (average annual mortality rate, $5.0 \%)[9]$.

Suspected CAD patients with chest symptom or abnormal result of electrocardiogram and echocardiogram should be performed exercise stress electrocardiogram for the diagnosis of ischemia if possible. CAG is performed in the high risk patients or patients diagnosed ischemia. Stress scintigram and/or cardiac CT are added in the moderate risk patients. As far as stress test, the patients who are unable to exercise sufficiently are selected drug stress test, although exercise stress test is 


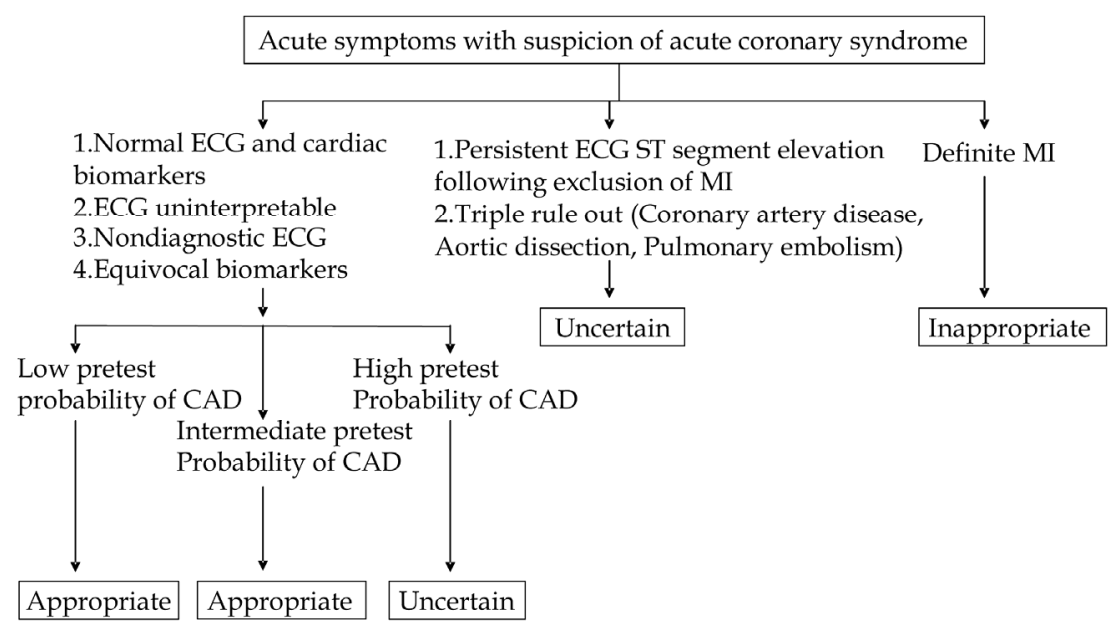

Figure 1. Detection of CAD in symptomatic patients without known heart disease [6].

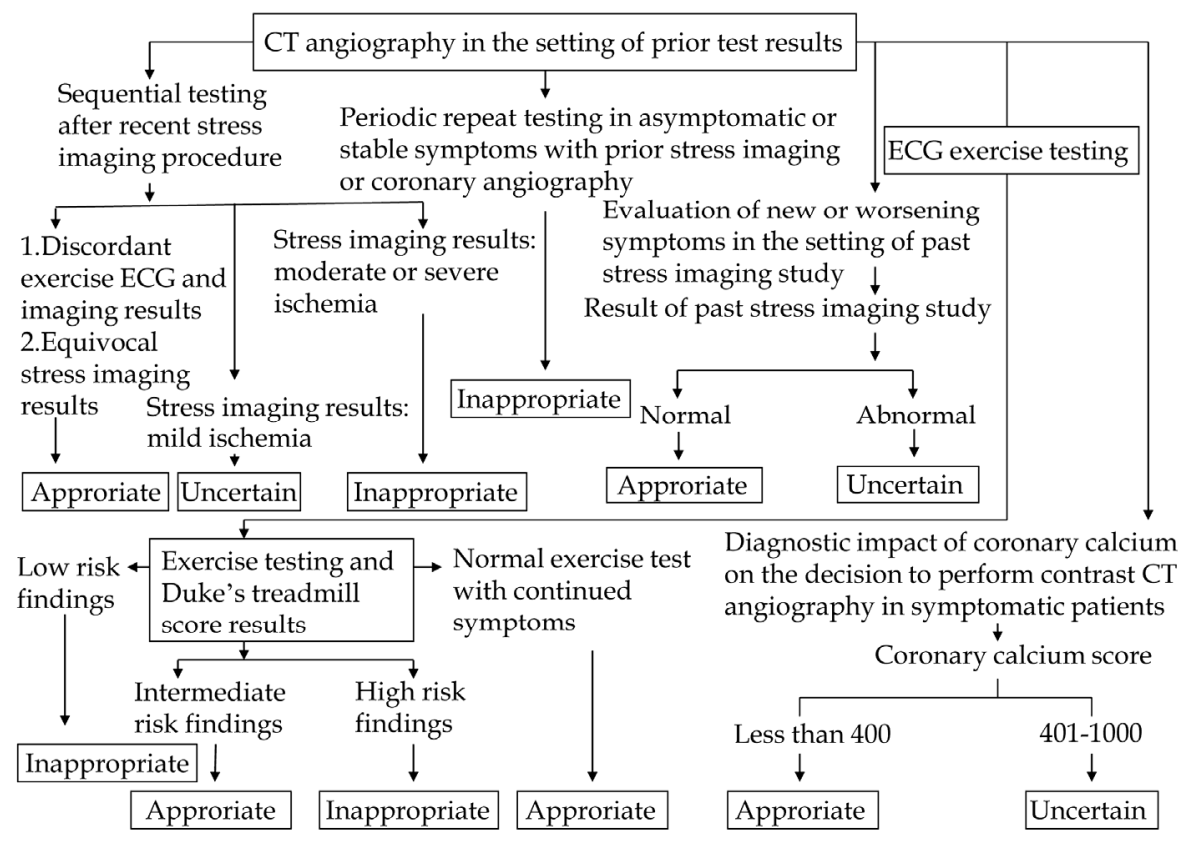

Figure 2. Use of CT angiography in the setting of prior test result [6].

Table 2. Pretest probability of CAD by age, sex, and symptom.

\begin{tabular}{|c|c|c|c|c|c|}
\hline Age & Sex & $\begin{array}{l}\text { Typical/definite } \\
\text { angina pectoris }\end{array}$ & $\begin{array}{l}\text { Atypical/probable } \\
\text { angina pectoris }\end{array}$ & Nonanginal chest pain & Asymptom \\
\hline \multirow{2}{*}{$30-39$} & Men & Intermediate & Intermediate & Low & Very low \\
\hline & Women & Intermediate & Very low & Very low & Very low \\
\hline \multirow{2}{*}{$40-49$} & Men & High & Intermediate & Intermediate & Low \\
\hline & Women & Intermediate & Low & Very low & Very low \\
\hline \multirow{2}{*}{$50-59$} & Men & High & Intermediate & Intermediate & Low \\
\hline & Women & Intermediate & Intermediate & Low & Very low \\
\hline \multirow{2}{*}{$60-69$} & Men & High & Intermediate & Intermediate & Low \\
\hline & Women & High & Intermediate & Intermediate & Low \\
\hline
\end{tabular}


more physiologic (Figures 3 and 4).

1) Hospital condition for cardiac CT

a) The staffs have sufficient experience on cardiac CT.

b) The hospital equips with cardiac CT which is better than 64 slice.

c) The staffs are able to show good images and work the proper reporting system.

d) The staffs understood the feature of each CAG and cardiac CT.

e) The staffs try to make the protocol for lowering the radiation exposure.

2) Patient condition for cardiac CT

a) The staffs are careful of the risk of radiation exposure in women which is less than 50 years old.

b) It is difficult to diagnose in the patients with many calcified coronary arteries, for example, the patients with hemodialysis, and the elderly patients.

c) Serum creatinin is less than $2.0 \mathrm{mg} / \mathrm{dL}$.

d) The eGFR is more than $60 \mathrm{~mL} / \mathrm{min} / 1.73 \mathrm{~m}^{2}$.

e) Diabetes mellitus nephropathy patients, including to microalbuminuria are not recommended.

f) The contrast alergy patients are not recommended.

g) Asthma patients are not recommended.

3) Stress SPECT

a) The stress test is recommended exercise stress, rather than drug stress.

b) The diagnosis is recommended 17 or 20 segment method to diagnose the area and degree of ischemia.

c) The staff confirms whether the patients have contraindication of the stress drug.

d) Echocardiogram and perfusion MRI can be alternative stress test depending on the facilities.

e) Less than $50 \%$ count of maximum count in the defect area is more than moderate ischemia.

f) The count between $50 \%$ and $70 \%$ count of maximum count in the defect area is mild ischemia.

g) More than $70 \%$ count of maximum count is normal myocardium.

\section{The Visualization of the Coronary Artery Lumen in Cardiac CT}

Cardiac CT can be applied for visualization of the coronary artery lumen after intravenous injection of a contrast agent. The administration of beta blockers before the cardiac CT scan and the use of sublingual nitroglycerin can achieve coronary vasodilation and maximize image quality. Studies using 64 slice CT scanning with invasive coronary angiography report sensitivities and specificities of $94 \%$ - 99\% and $86 \%$ - 97\%, respectively, and importantly, a negative predictive value of $98 \%-100 \%$ [10-12]. Patency and occlusion of bypass grafts can be established with very high accuracy in cardiac CT [13, 14]. But, the limitations are the detection of stenoses at

Suspected CAD patients with chest symptom, or abnormal results of rest electrocardiogram and echocardiogram.

The patients can be performed exercise electrocardiogram with sufficient stress exercise. The patients' ischemic heart disease can be diagnosed with electrocardiogram. $\rightarrow$ Figure 4
No $\downarrow$ Yes

Exercise stress electrocardiogram, Duke's treadmill ECG score

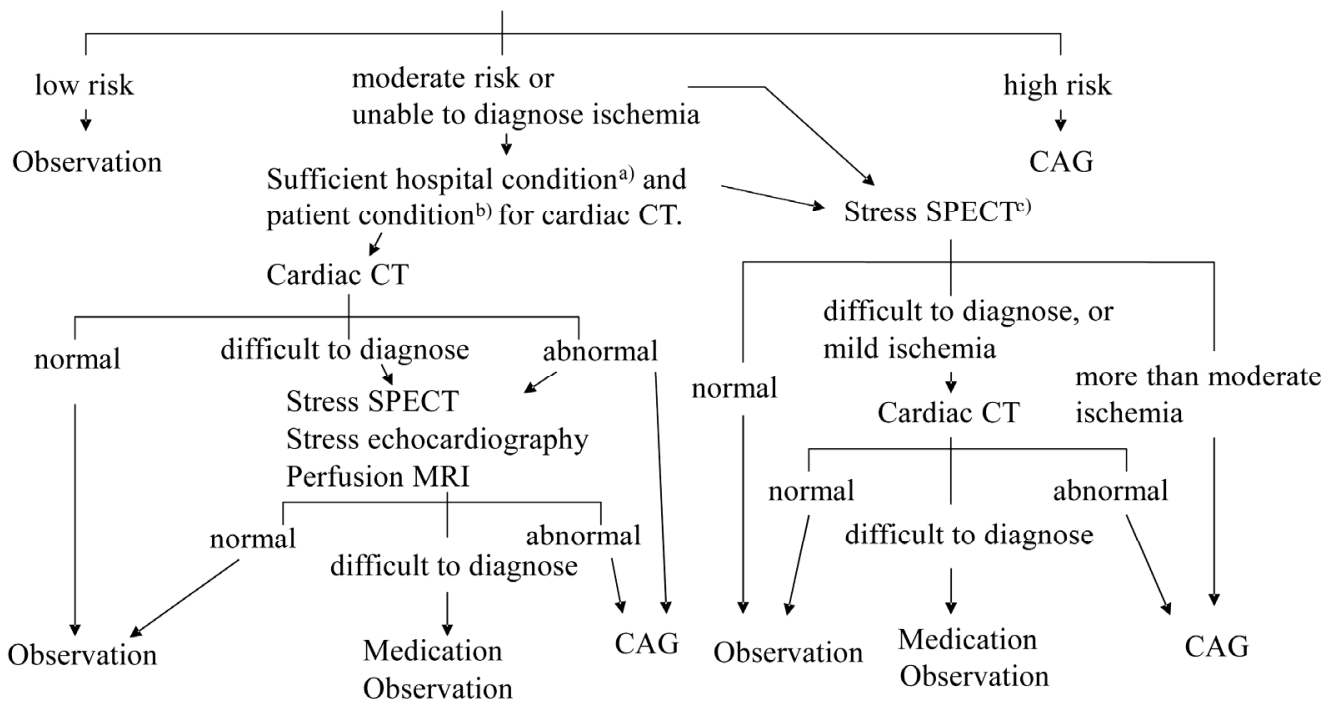

Figure 3. Algorithm to diagnose stable angina pectoris for the patients who are able to exercise (revised Japanese Circulation Society Guideline 2009, Circulation Journal, Vol. 73, Suppl. III, 2009, pp. 1019-1089). 


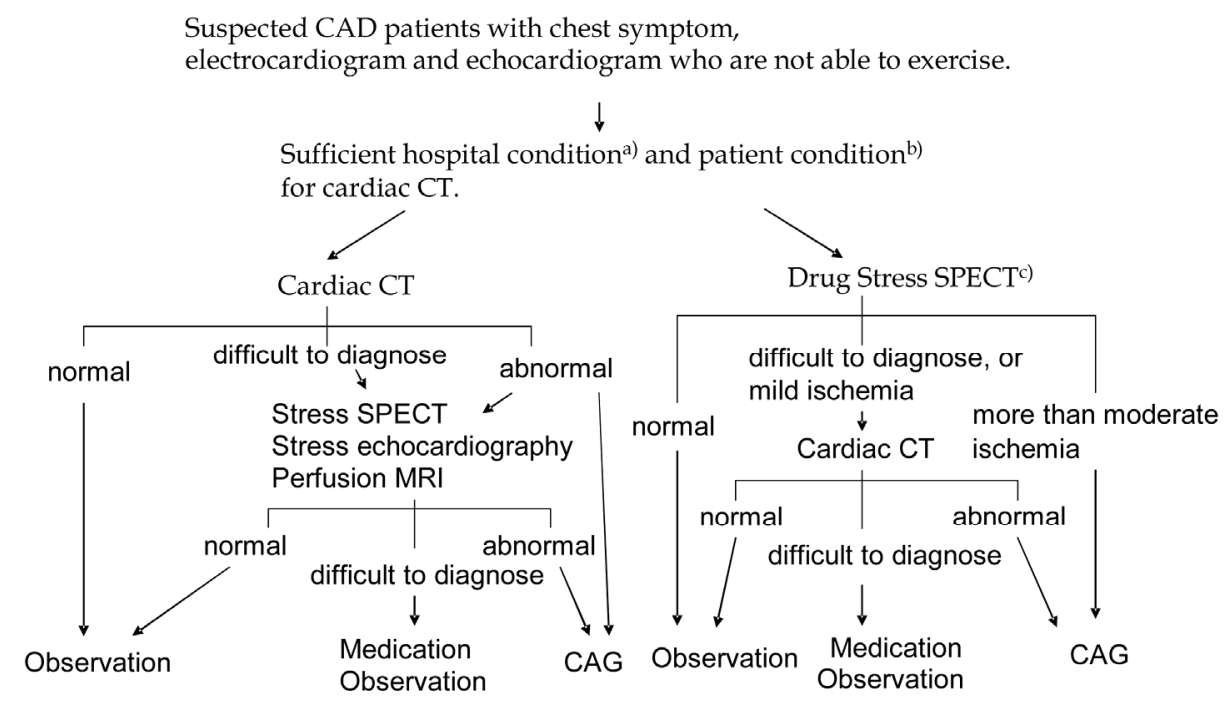

Figure 4. Algorithm to diagnose stable angina pectoris for the patients who are not able to exercise (revised Japanese Circulation Society Guideline 2009, Circulation Journal, Vol. 73, Suppl. III, 2009, pp. 1019-1089).

the site of anastomosis to the coronary artery and in the peripheral run-off coronary artery. Cardiac CT with volume rendering images is useful to patients with known or suspected congenital coronary artery anomalies. Cardiac $\mathrm{CT}$ can classify the origin and the complex course of anomalous coronary arteries $[15,16]$.

Although artifacts caused by metal and partial volume effects limit the evaluation of in-stent restenosis with 64-slice CT, the sensitivity was $91 \%$, specificity was $91 \%$, positive predictive value was $68 \%$, and negative predictive value was $98 \%$ [9].

It is important to note that an analysis of the degree of coronary stenosis and risk areas of the myocardium can be performed without additional radiation exposure or contrast injections. The introduction of myocardial assessment by first-pass scan protocols into clinical practice is easy without additional stress and cost.

\section{The Morphology of Atherosclerosis in the Coronary Artery}

Atherosclerosis consists of two pathological processes. Atherosis characterized by morphologic atheromatous lesions appearing in the intima, and sclerosis characterized by an increase in stiffness of the vessel walls.

Cardiac CT has several potential applications for patients with coronary artery disease. Cardiac CT can demonstrate the morphological consequences of coronary artery disease and estimate ventricular function, perfusion. Recently, the visualization of coronary artery has been developing with coronary artery calcification, coronary artery stenosis.

Non-enhanced CT studies of the heart are almost exclusively performed to assess calcified structures within the heart and coronary artery calcification (CAC).
As far as the pathology of coronary artery, CAC is a surrogate marker for coronary atherosclerotic plaque. In the coronary arteries, calcifications occur almost exclusively in the context of atherosclerotic changes $[17,18]$. In the most patients with acute coronary syndromes, CAC can be detected, and the amount of calcium in these patients is substantially greater than in the patients without coronary artery disease [19]. With the exception of patients in the renal failure, nonatherosclerotic calcification of the coronary artery wall is rare. CAC is not associated with stability or instability of an individual plaque [18]. Plaques with healed ruptures contain calcium, whereas plaque erosions are frequently not calcified.

Calcification does not occur in a normal vessel wall, it therefore indicates the presence of atherosclerosis, and endothelial dysfunction in the coronary arteries but is not specific for luminal obstruction.

However, the absence of detectable CAC rules out the presence of significant coronary artery stenoses with high negative predictive value [17]. Because even coronary atherosclerotic plaque burden is not necessarily associated with hemodynamically luminal narrowing, even the detection of large amounts of calcium does not imply the presence of the significant stenoses. The greatest potential for CAC detection could be as a marker for CAD prognosis in asymptomatic patients. The presence and severity of CAC has independent and incremental value in the estimation of death or nonfatal MI [20]. Coronary calcium is important in the risk stratification, and noncalcified atherosclerosis is also important. Some studies against intravascular ultrasound have reported sensitivities of 53 to 83 percent for the detection of noncalcified plaque by 16 and 64 slice CT [21-23]. Noncalcified plaque is consisted of lipid-rich plaques and fibrous plaques. 
Lipid-rich plaque (14 to $72 \mathrm{HU}$ ) is lower CT Hounsfield attenuation numbers than fibrous plaques (90 to $121 \mathrm{HU}$ ) $[23,24]$. Patients with acute coronary syndromes were found to have a higher prevalence of noncalcified as compared with calcified plaque than stable coronary artery disease [25].

\section{Conclusion}

Cardiac CT is a rapidly developing and advancing technology. The diagnostic accuracy of Cardiac CT in the detection of coronary artery stenoses has been reported with very high negative predictive values by many investigators. The increase of radiation will reduce artifact, improve coronary artery visualization, although an excess of radiation exposure leads to cancer. Cardiac CT is useful in the invasive diagnosis of coronary artery disease for the stable and acute chest pain patients, especially who cannot be sufficiently evaluated by electrocardiography, echocardiography or cardiac MRI.

\section{REFERENCES}

[1] R. O. Bonow, L. A. Smaha, S. C. Smith Jr., G. A. Mensah and C. Lenfant, "World Heart Day 2002: The International Burden of Cardiovascular Disease: Responding to the Emerging Global Epidemic," Circulation, Vol. 106, No. 13, 2002, pp. 1602-1605. doi:10.1161/01.CIR.0000035036.22612.2B

[2] T. M. Bashore, E. R. Bates, P. B. Berger, et al., "American College of Cardiology/Society for Cardiac Angiography and Interventions Clinical Expert Consensus Document on Cardiac Catheterization Laboratory Standards. A Report of the American College of Cardiology Task Force on Clinical Expert Consensus Documents," Journal of the American College of Cardiology, Vol. 37, No. 8, 2001, pp. 2170-2214. doi:10.1016/S0735-1097(01)01346-8

[3] G. N. Hounsfield, "Computerized Transverse Axial Scanning (Tomography). 1. Description of System," British Journal of Radiology, Vol. 46, No. 552, 1973, pp. 10161022. doi:10.1259/0007-1285-46-552-1016

[4] U. Hoffmann, J. T. Nagurney, F. Moselewski, et al., "Coronary Multidetector Computed Tomography in the Assessment of Patients with Acute Chest Pain," Circulation, Vol. 114, No. 21, 2006, pp. 2251-2260. doi:10.1161/CIRCULATIONAHA.106.634808

[5] A. J. Taylor, M. Cerqueira, J. M. Hodgson, et al., "ACCF/SCCT/ACR/AHA/ASE/ASNC/NASCI/SCAI/SC MR 2010 Appropriate Use Criteria for Cardiac Computed Tomography: A Report of the American College of Cardiology Foundation Appropriate Use Criteria Task Force, the Society of Cardiovascular Computed Tomography, the American College of Radiology, the American Heart Association, the American Society of Echocardiography, the American Society of Nuclear Cardiology, the North American Society for Cardiovascular Imaging, the Society for Cardiovascular Angiography and Interventions, and the Society for Cardiovascular Magnetic Resonance," Circulation, Vol. 122, No. 21, 2010, pp. e525-e555.

doi:10.1161/CIR.0b013e3181fcae66

[6] S. B. King III, S. C. Smith Jr, J. W. Hirshfeld Jr., A. K. Jacobs, D. A. Page, B. Riegel, L. G. Tarkington, C. W. Y. Hunt, H. M. Krumholz, F. G. Kushner, B. W. Lytle, R. Nishimura, R. L. Christopher, E. Buller, M. A. Creager, S. M. Ettinger, J. L. Halperin, S. A. O. Williams, S. C. Smith Jr., A. K. Jacobs, C. D. Adams, J. L. Anderson III, D. A. Morrison, W. W. O'Neill, H. V. Schaff, P. L. Whitlow, T. E. Feldman, J. W. Hirshfeld Jr, A. K. Jacobs, M. J. Kern, S. B. K. Morrison, D. O. Williams and S. C. Smith (2005 Writing Committee Members), "2007 Focused Update of the ACC/AHA/SCAI 2005 Guideline Update for Percutaneous Coronary Intervention, Writing on Behalf of the 2005 Writing Committee Evidence and Update the ACC/AHA/SCAI 2005 Guideline Update for Percutaneous Association Task Force on Practice Guidelines: 2007 Writing Group to Review New Coronary Intervention : A Report of the American College of Cardiology/American Heart," Circulation, Vol. 117, No. 2, 2008, pp. 261-295.

[7] G. A. Diamond and J. S. Forrester, "Analysis of Probability as an Aid in the Clinical Diagnosis of Coronary Artery Diseases," New England Journal of Medicine, Vol. 300, 1979, pp. 1310-1358. doi:10.1056/NEJM197906143002402

[8] G. A. Diamond, J. S. Forrester and M. Hirsch, "Application of Conditional Probability Analysis to the Clinical Diagnosis of Coronary Artery Disease," Journal of Clinical Investigation, Vol. 65, No. 5, 1980, pp. 1210-1221. doi:10.1172/JCI109776

[9] D. J. Kumbhani, C. P. Ingelmo, P. Schoenhagen, R. J. Curtin, S. D. Flamm and M. Y. Desai, "Meta-Analysis of Diagnostic Efficacy of 64-Slice Computed Tomography in the Evaluation of Coronary In-Stent Restenosis," can Journal of Cardiology, Vol. 103, No. 12, 2009, pp. 16751681. doi:10.1016/j.amjcard.2009.02.024

[10] S. Leschka, H. Alkadhi, A. Plass, et al., "Accuracy of MSCT Coronary Angiography with 64-Slice Technology: First Experience," European Heart Journal, Vol. 26, No. 15, 2005, pp. 1482-1487. doi:10.1093/eurheartj/ehi261

[11] N. R. Mollet, F. Cademartiri, C. A. van Mieghem, et al., "High-Resolution Spiral Computed Tomography Coronary Angiography in Patients Referred for Diagnostic Conventional Coronary Angiography," Circulation, Vol. 112, No. 15, 2005, pp. 2318-2323. doi:10.1161/CIRCULATIONAHA.105.533471

[12] G. L. Raff, M. J. Gallagher, W. W. O’Neill and J. A. Goldstein, "Diagnostic Accuracy of Noninvasive Coronary Angiography Using 64-Slice Spiral Computed Tomography," Journal of the American College of Cardiology, Vol. 46, No. 3, 2005, pp. 552-557. doi:10.1016/j.jacc.2005.05.056

[13] J. M. Miller, C. E. Rochitte, M. Dewey, et al., "Diagnostic Performance of Coronary Angiography by 64-Row CT," New England Journal of Medicine, Vol. 359, No. 22, 2008, pp. 2324-2336. doi:10.1056/NEJMoa0806576

[14] M. J. Budoff, D. Dowe, J. G. Jollis, et al., "Diagnostic Performance of 64-Multidetector Row Coronary Com- 
puted Tomographic Angiography for Evaluation of Coronary Artery Stenosis in Individuals without Known Coronary Artery Disease: Results from the Prospective Multicenter ACCURACY (Assessment by Coronary Computed Tomographic Angiography of Individuals Undergoing Invasive Coronary Angiography) Trial," Journal of the American College of Cardiology, Vol. 52, No. 21, 2008, pp. 1724-1732. doi:10.1016/j.jacc.2008.07.031

[15] J. Datta, C. S. White, R. C. Gilkeson, et al., "Anomalous Coronary Arteries in Adults: Depiction at Multi-Detector Row CT Angiography," Radiology, Vol. 235, No. 3, 2005, pp. 812-818. doi:10.1148/radiol.2353040314

[16] M. Schmid, S. Achenbach, J. Ludwig, et al., "Visualization of Coronary Artery Anomalies by Contrast-Enhanced Multi-Detector Row Spiral Computed Tomography," International Journal of Cardiology, Vol. 111, No. 3, 2006, pp. 430-435. doi:10.1016/j.ijcard.2005.08.027

[17] M. J. Budoff, S. Achenbach, R. S. Blumenthal, et al., "Assessment of Coronary Artery Disease by Cardiac Computed Tomography: A Scientific Statement from the American Heart Association Committee on Cardiovascular Imaging and Intervention, Council on Cardiovascular Radiology and Intervention, and Committee on Cardiac Imaging, Council on Clinical Cardiology," Circulation, Vol. 114, No. 16, 2006, pp. 1761-1791. doi:10.1161/CIRCULATIONAHA.106.178458

[18] A. P. Burke, R. Virmani, Z. Galis, C. C. Haudenschild and J. E. Muller, "34th Bethesda Conference: Task Force \#2-What Is the Pathologic Basis for New Atherosclerosis Imaging Techniques?" Journal of the American College of Cardiology, Vol. 41, No. 11, 2003, pp. 1874-1886. doi:10.1016/S0735-1097(03)00359-0

[19] K. Pohle, D. Ropers, R. Maffert, et al., "Coronary Calcifications in Young Patients with First, Unheralded Myocardial Infarction: A Risk Factor Matched Analysis by Electron Beam Tomography," Heart, Vol. 89, No. 6, 2003, pp. 625-658. doi:10.1136/heart.89.6.625

[20] P. G. O’Malley, A. J. Taylor, J. L. Jackson, T. M. Do- herty and R. C. Detrano, "Prognostic Value of Coronary Electron-Beam Computed Tomography for Coronary Heart Disease Events in Asymptomatic Populations," American Journal of Cardiology, Vol. 85, No. 8, 2000, pp. 945-948. doi:10.1016/S0002-9149(99)00906-6

[21] S. Achenbach, F. Moselewski, D. Ropers, et al., "Detection of Calcified and Noncalcified Coronary Atherosclerotic Plaque by Contrast-Enhanced, Submillimeter Multidetector Spiral Computed Tomography: A SegmentBased Comparison with Intravascular Ultrasound," Circulation, Vol. 109, No. 1, 2004, pp. 14-17. doi:10.1161/01.CIR.0000111517.69230.0F

[22] A. W. Leber, A. Becker, A. Knez, et al., "Accuracy of 64-Slice Computed Tomography to Classify and Quantify Plaque Volumes in the Proximal Coronary System: A Comparative Study Using Intravascular Ultrasound," Journal of the American College of Cardiology, Vol. 47, No. 3, 2006, pp. 672-677. doi:10.1016/j.jacc.2005.10.058

[23] A. W. Leber, A. Knez, A. Becker, et al., "Accuracy of Multidetector Spiral Computed Tomography in Identifying and Differentiating the Composition of Coronary Atherosclerotic Plaques: A Comparative Study with Intracoronary Ultrasound," Journal of the American College of Cardiology, Vol. 43, No. 7, No. 2004, pp. 12411247.

[24] K. Pohle, S. Achenbach, B. Macneill, et al., "Characterization of Non-Calcified Coronary Atherosclerotic Plaque by Multi-Detector Row CT: Comparison to IVUS," Atherosclerosis, Vol. 190, No. 1, 2007, pp. 174-180. doi:10.1016/j.atherosclerosis.2006.01.013

[25] U. Hoffmann, F. Moselewski, K. Nieman, et al., "Noninvasive Assessment of Plaque Morphology and Composition in Culprit and Stable Lesions in Acute Coronary Syndrome and Stable Lesions in Stable Angina by Multidetector Computed Tomography," Journal of the American College of Cardiology, Vol. 47, No. 8, 2006, pp. 1655-1662. doi:10.1016/j.jacc.2006.01.041 